\title{
Overshadowing depends on cue and reinforcement sensitivity but not schizotypy
}

Clare Pickett, Helen J. Cassaday*, Peter A. Bibby

School of Psychology

University of Nottingham, United Kingdom

*Correspondence:

Helen Cassaday

School of Psychology

University of Nottingham

University Park

Nottingham

NG7 2RD

United Kingdom

email: helen.cassaday@nottingham.ac.uk

Running head:

Overshadowing and cue sensitivity

Key words:

Overshadowing; associative learning; task motivation; schizotypy; reinforcement sensitivity 


\begin{abstract}
There is evidence for impaired selective learning mechanisms in individuals high in schizotypy. Overshadowing provides a direct test of selective learning based on cue salience and has previously been reported to be impaired in relation to schizotypy scores. The present study tested for overshadowing using food allergy and Lego construction task variants. Both variants used the same number of conditioned stimulus (CS) cues and the same number of learning trials. CS cues were trained in compound pairs or in isolation and overshadowing was subsequently tested on trials followed by negative versus positive outcomes. Participants also completed the O-LIFE to measure schizotypy and BIS-BAS scales to measure reinforcement sensitivity. Learning was demonstrated for both cue variants; however overshadowing emerged only in the Lego variant and only on the trials followed by the negative outcome. Contrary to expectations, there was no evidence for any relationship between overshadowing and O-LIFE scores. However, there was evidence of a positive relationship between overshadowing and BAS-Drive as well as a negative relationship with BIS-Anxiety, for the trials followed by the positive outcome in the food allergy variant. These results suggest that the development of overshadowing depends on cue and reinforcement sensitivity, but not necessarily on schizotypy.
\end{abstract}




\section{Introduction}

Learning causal relationships between environmental cues and outcomes is a fundamental learning ability. Not only does it enable humans and other animals to anticipate and thus potentially control events likely to occur, selective learning provides a filter on impinging sensory impressions and cues competing for attention. Thus, associative learning is restricted to the best predictors of outcomes, in order to represent the causal structure of the environment.

Latent inhibition (LI) [1] and Kamin blocking (KB) [2] are two-stage procedures whereby learning in stage 1 reduces learning in stage 2, because of the perceived irrelevance of a pre-exposed cue in LI or the redundancy of an additional cue in blocking. Both of these learning phenomena demonstrate how past experience modulates the salience of a stimulus; cues become less salient and therefore less attention is paid to them as a result of previous learning ('acquired' salience, [3]). Over and above differences in salience in consequence of past experience with a cue, inherent physical features (e.g., colour, size, intensity) of a stimulus determine its 'intrinsic' salience [3]. Effects of intrinsic salience on selective learning are demonstrated using overshadowing (OS) procedures [4]. OS describes how learning about one stimulus (A) is reduced when it is paired in compound with a more intense second stimulus (B), as compared to the trials where that same stimulus (A) is presented alone. Thus, cues in compound compete based on relative intensity; typically a more intense stimulus acquires associative strength at the expense of that accrued to a less intense stimulus. This effect of cue intensity has been formally incorporated within the attentional parameters of theories of associative learning theory $[5,6,7]$ : the more salient stimulus captures more attention and thus provides a more effective predictor of the outcome.

Both LI and KB have been found to be dysfunctional in individuals with schizophrenia and this impairment in selectivity of learning has been argued to provide some account of their cognitive symptoms [8-14]. Indeed it has long been suggested that the inability to filter or tune out irrelevant stimuli is a hallmark symptom of schizophrenia [15]. As demonstrated by evidence of disrupted LI and KB in acute patients, they may be 'overlearning' about cues in their environment which have previously been without consequence (LI) or redundant (KB). Deficiencies in LI and/or KB are said to reflect 'hyperassociability', whereby individuals form associations about cues which erroneously appear as salient, consistent with more recent theories proposing that psychosis can be 
understood in terms of aberrant salience processing [16-18].

In common with $\mathrm{LI}$ and $\mathrm{KB}$, in rat studies, OS has been reported to be disrupted by amphetamine, an indirect dopamine agonist known to produce psychotic-like symptoms in non-schizophrenic samples. Following drug administration, the previously salient stimulus no longer overshadowed the less salient in the compound $[19,20]$. However, there is evidence to suggest that the effective dose is different from that sufficient to disrupt LI [21] and - to date there are no reports of differences in OS in schizophrenia patients or non-schizophrenic drugtreated human populations and few studies of individual differences within the normal range.

Schizophrenia and schizophrenic-like traits represent a complex and heterogeneous phenotype which has been argued to be continuously rather than dichotomously distributed [22-25; but see 26]. Thus, subclinical signs and symptoms of schizophrenia are observed in otherwise healthy individuals and are part of normal behaviour and experience. The OxfordLiverpool Inventory of Feelings and Emotions (O-LIFE) [22-24] was devised to measure four dimensions of schizotypy intended to reflect schizophrenic symptoms: unusual experiences (UnExp), cognitive disorganisation (CogDis), introvertive anhedonia (IntAn) and impulsive nonconformity (ImpNo). Many cognitive processes which are dysfunctional in schizophrenia sufferers are similarly dysfunctional in individuals who score highly on schizotypy scales $[24,27]$. For example, there is good evidence that reduced LI may be attributed to high schizotypy, as measured by a variety of scales [8]. In particular, studies using the O-LIFE show that increased UnExp scores are correlated with reduced LI [28, 29]. Reduced LI has also been reported in individuals with increased CogDis and ImpNo scores [29]. Similarly, there is evidence for reduced $\mathrm{KB}$ in individuals scoring highly on UnExp and CogDis schizotypy measures $[13,30]$. Using a geometric associative learning task, Granger, Prados and Young [31] demonstrated attenuated LI and OS in high-schizotypy individuals, specifically relating reduced OS and LI to increased UnExp scores. However, task variant may be a critical factor since a later study which used a different (letter identification) task variant reported the opposite direction of association for LI [32]. OS is a standard control in KB designs. Thus, (individual differences in) the reliability with which OS may be demonstrated is a likely confound of (individual differences in) KB. However, there been relatively little systematic investigation of the brain substrates of OS and how these may compare with those of KB. Moreover, if different mechanisms are responsible for LI, KB and OS, then we would expect OS to show a different relation with schizotypy scores to that reported to date [31]. 
Learning about environmental cues is also determined by individual sensitivity to reward, non-reward and punishment. Gray's Reinforcement Sensitivity Theory (RST) [33-35] is a biologically determined personality theory which relates the regulation of behaviour and affect to (participants' sensitivity to) motivationally significant environmental stimuli. Carver and White's [36] behavioural inhibition (BIS) and activation system (BAS) scales are the most widely used measure of the original RST constructs. As per the early versions of RST [35], BAS is held to be responsive to all appetitive and rewarding stimuli and to mediate approach behaviour, related to impulsivity as defined by other measures [37]. In more recent developments, the RST revised form [38] posits a third underlying construct, the FightFlight-Freeze System (FFFS). FFFS activation results in fear, facilitates the avoidance of aversive stimuli, and subsumes the sensitivity to punishment or non-reward which was previously the role of the BIS. Rather, the BIS is postulated responsible for resolution of goal conflict, for example in situations when both the BAS (approach) and the FFFS (avoidance) have been activated [38]. Based on an earlier study of human associative learning, BAS scores would be predicted to relate to reduced excitatory conditioning [39]. Any such effect would be expected to influence the level of OS.

Accordingly, the present study used two associative learning task variants to examine the relationship between OS performance, schizotypy and BIS-BAS scores. Food allergy paradigms are commonly adopted to provide a Pavlovian type procedure [40]. However, for the majority of participants, a variety of food cues will not be treated as neutral to begin with and moreover, it has been argued that such contingency judgements depend on higher cognitive processes such as reasoning and propositional knowledge [41, 42]. Therefore the present study tested OS using both a food allergy paradigm and a novel but analogous task conducted using neutral (Lego) cues, to allow examination of how levels of associative learning and OS seen in the two task variants, conducted under otherwise identical conditions, would be affected by the nature of the cues. In the food task, the CS cues would be easily identifiable and potentially have pre-formed associations likely to facilitate (or limit) learning new associations. However, in the Lego task, the novel CS images would likely have no pre-formed associations and thus any learning would only arise in consequence of the CS-US pairings.

The hypotheses under test were that (1) levels of learning and OS would depend on the nature of the CS and task variant in use; (2) OS would be attenuated in participants with higher O-LIFE scores, specifically on the UnExp sub-scale; and (3) reinforcement sensitivity 
as measured by the BIS-BAS would relate to levels of associative learning.

\section{Method}

\subsection{Participants}

This study used a sample of 66 participants (30 males and 36 females) of mean age (SD; range) 32.6 (15; 20-79) years. All of the participants had completed secondary school, 11 were undergraduates, 41 were postgraduates and 10 were undergoing or had completed some further professional training.

The study was approved by the University of Nottingham School of Psychology Ethics Committee. As compensation for any inconvenience, all participants were entered into a prize draw with the chance to win one of ten $£ 10$ gift vouchers.

\subsection{Stimuli and materials}

2.2.1. Computer-based tasks. Lego cues were provided by photos of coloured blocks and pieces in distinct configurations (Figure 1). Outcomes were presented on a separate screen as 'Collapsed!' in red text or 'Didn't collapse!' in green text. The food cues were selected from images freely available from the internet (e.g. chicken, broccoli, garlic cloves, egg; not reproduced here). The food-types chosen were similar to those used previously in the food allergy literature [43-45]. Outcomes were presented on a separate screen as 'Food allergy!' in red text or 'No food allergy!' in green text.

The learning tasks were programmed using PsychoPy2 [46]. For the food variant, participants were required to associate a food with the outcome of allergy or no allergy; foods were presented alone or in compound. Participants were requested to imagine that they are a food allergy tester: "You will be shown pictures of food. You will then be told if these foods led to a food allergy or not. You must learn which foods will lead to a food allergy." For the Lego variant, participants were required to associate a Lego configuration with the outcome of collapse or no collapse; Lego images were presented alone or in compound. Participants were requested to imagine that they work for a Lego construction company: "You have begun to build, however some of the pieces are faulty. As a result, some of the constructions have collapsed. You must remember which constructions have collapsed or not." Depending on the availability of the participants, the tasks were presented on an Apple desktop computer (21.5" 
screen) or a DELL laptop (15.4" screen). For each participant, both tasks were presented using the same device and screen.

\section{Figure 1}

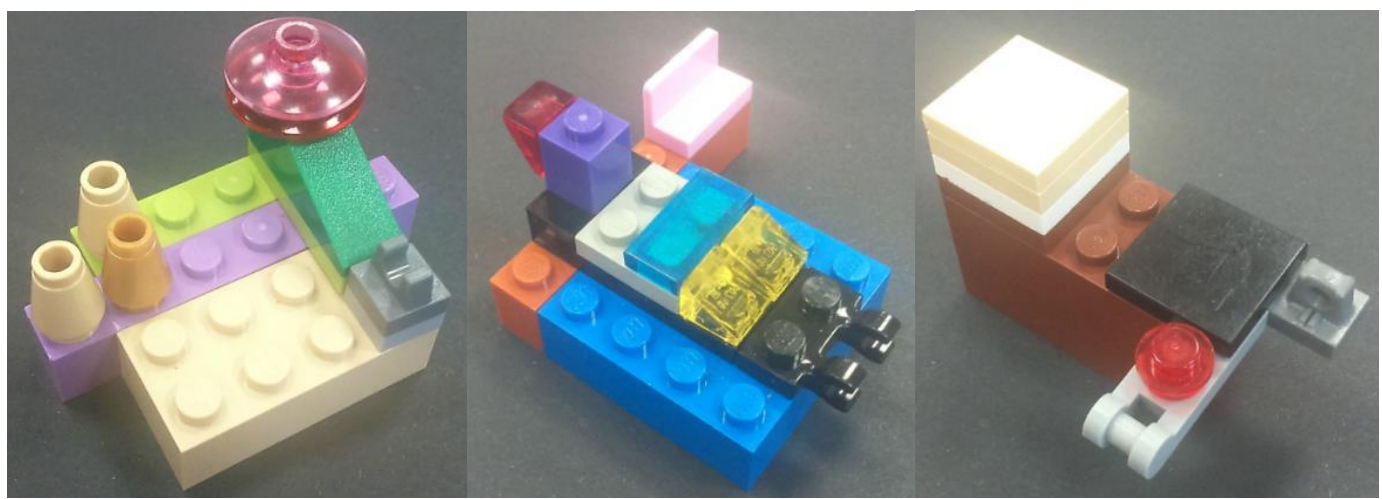

Note: Example Lego stimuli used as conditioned stimuli in the second task variant.

2.2.2 Questionnaires. The O-LIFE short form [24] is a 43-item dichotomous questionnaire which measures schizotypy across four sub-scales: UnExp (12 items), CogDis (11 items), IntAn (10 items) and ImpNo (10 items). The BIS/BAS scale [36] is a 24-item questionnaire which measures BAS under three sub-scales, BAS-Reward Responsiveness (5 items), BAS-Fun-seeking (4 items) and BAS-Drive (4 items), BIS-anxiety (4 items) and BISFFFS (3 items). The scale also includes 4 filler items. Responses are made on a 4-point scale from $1=$ very true for me to $4=$ not true for me. For the majority of items, lower scores represented a higher sensitivity so these items were reverse scored to ease data interpretation.

\subsection{Procedure}

Participants were allocated one of six counterbalanced task pair variants and requested to complete two learning tasks involving associating images with outcomes, each comprised of two stages: a learning stage and a test stage. More detailed instructions were subsequently given on screen for both tasks. Participants were given the opportunity to ask any questions before the learning trials began. The two tasks used the same number of CSs and trials as well as the same on screen presentation durations. In learning trials, CSs (presented alone or in compound) appeared on screen for $2.5 \mathrm{~s}$ and were immediately followed by the outcome screen which appeared for 2.0s. There was an inter-trial-interval (1.5s) between each trial presentation. There were $12 \mathrm{CSs}$, four pairs for the compound condition and four in the single condition. In each block there were 16 trials in total (see 
Table 1 for experimental design). Each block of trials was repeated four times (64 trials in total). This meant that each compound or single stimulus appeared eight times. Presentation across each block of trials was randomised. At test, each stimulus was presented alone with a Likert rating scale of 1-9 with no time limit. In the Lego task, participants were asked 'How likely is it that this construction will collapse?' (1=unlikely, 5=unsure, 9=very likely). Similarly in the food allergy task they were asked 'How likely is it that this food will lead to an allergy?' (1=unlikely, 5=unsure, $9=$ =ery likely). Participants indicated their ratings using the number keys. Each stimulus was presented twice (in a random sequence) for a more reliable test rating. The two tasks took altogether approximately 15 min to complete.

After finishing both tasks, participants completed the two questionnaires.

\section{Table 1}

Experimental design

\begin{tabular}{|llll|}
\hline Condition & Presentation & Learning stage & Test stage \\
1 & Comp-ve & AE-Collapsed! & A? E? \\
2 & Comp+ve & CG-Didn't collapse! & C? G? \\
3 & Sing-ve & J-Collapsed! & J? \\
4 & Sing+ve & L- Didn't collapse! & L? \\
& & & \\
\hline
\end{tabular}

Note: Letters refer to individual Lego configurations or food images and denote example presentations used within the different experimental conditions. There were 4 experimental conditions per task each of which used four different configurations of stimuli, counterbalanced in regards to position on the screen. Images were followed by the presence of a contingent negative outcome (-ve) ('Collapsed!'; 'Food allergy!') or followed by the absence of a contingent positive 'omitted' outcome (+ve) ('Didn't collapse!; 'No food allergy!'). In total 16 trial types were randomly presented and repeated four times. At test, individual images were rated twice.

\subsection{Design and analysis}

For counterbalancing, three cue variants of each task were used, in order that each stimulus was either presented both in compound and alone (across the different versions). The positioning of the stimuli for both the compound and single presentations was also counterbalanced over trials, so that each stimulus appeared an equivalent number of times on the left versus on the right. The order of tasks was reversed for half the participants so there 
were six task pair variants in total: both Food-Lego and Lego-Food versions.

Factorial analysis was used to test for OS in the two task variants. The dependent variable (DV) entered into ANOVA was the participants' $(n=66)$ response ratings at the test stage for each stimulus in both tasks. Responses were coded according to whether they were followed by a negative (i.e. food allergy or collapsed) versus a positive 'omitted' outcome (i.e. no food allergy or did not collapse). Each of the 12 stimuli was tested twice. Eight of the stimuli had appeared during the learning stage in compound and four appeared alone. Preliminary analyses showed no systematic effect of stimulus identity confirming that the food and Lego cues were generally well-matched prior to conditioning. As there was an uneven number of items in the presentation condition (compound, single) average ratings were calculated over stimuli and repetition. These average ratings were used in a three-way $(2 \times 2 \times 2)$ repeated measures ANOVA with factors cue [Lego, food] $x$ outcome [negative, positive] x presentation [compound, single]. Interactions involving outcome and presentation are required to confirm OS in relation to the designated outcomes.

Correlational analyses were conducted using a 2-tailed Pearson's $r$ correlation, with an unadjusted alpha of $p \leq .05$ for exploratory purposes, to assess whether the level of OS was related to the measures of individual differences. For the purpose of the correlational analyses, summary learning scores were calculated for each participant. These were the mean difference scores between the singly and compound trained, negative versus positive cues, for each task variant.

\section{Results}

\subsection{Factorial analysis: learning and $\mathrm{OS}$ in the two task variants}

There was an effect of task, $F(1,65)=10.349, p=.002, M S e=4.833, \eta_{i}^{\prime:}=.137$. Participants rated stimuli higher in the Lego $(M=5.073)$ than the food task variant $(M=4.766)$, overall across both negative and positive cues. There was an effect of outcome, $F(1,65)=1109.316, p<.001, M S e=15.131, \eta_{i}^{\ddot{i}}=.945$. As would be expected given the configuration of the rating scale, participants rated the negative higher $(M=7.739)$ than the positive cues $(M=2.100)$. Consistent with an OS effect, there was an effect of presentation, $F(1,65)=9.317, p=.003, M S e=3.760, n_{i:}^{\ddot{i}}=.125$. Participants rated stimuli presented in compound overall lower $(M=4.791)$ than stimuli which had been presented alone $(M=5.048)$. 
There was a significant two-way interaction between outcome and presentation, $F(1,65)=16.813, p<.001, M S e=1.933, r_{i=}^{\ddot{z}}=.206$. The simple effects of outcome were significant for both compound and single presentations $(p<.001)$. In both cases the mean ratings for negative was greater than for positive cues. The simple effect of presentation for negative cues was significant $(p<.001)$ with mean ratings being greater for single than compound presentations, but the simple effect of presentation for positive cues was not significant $(p=.094)$. Thus, there is evidence of OS for negative but not for positive cues.

\section{Figure 2}

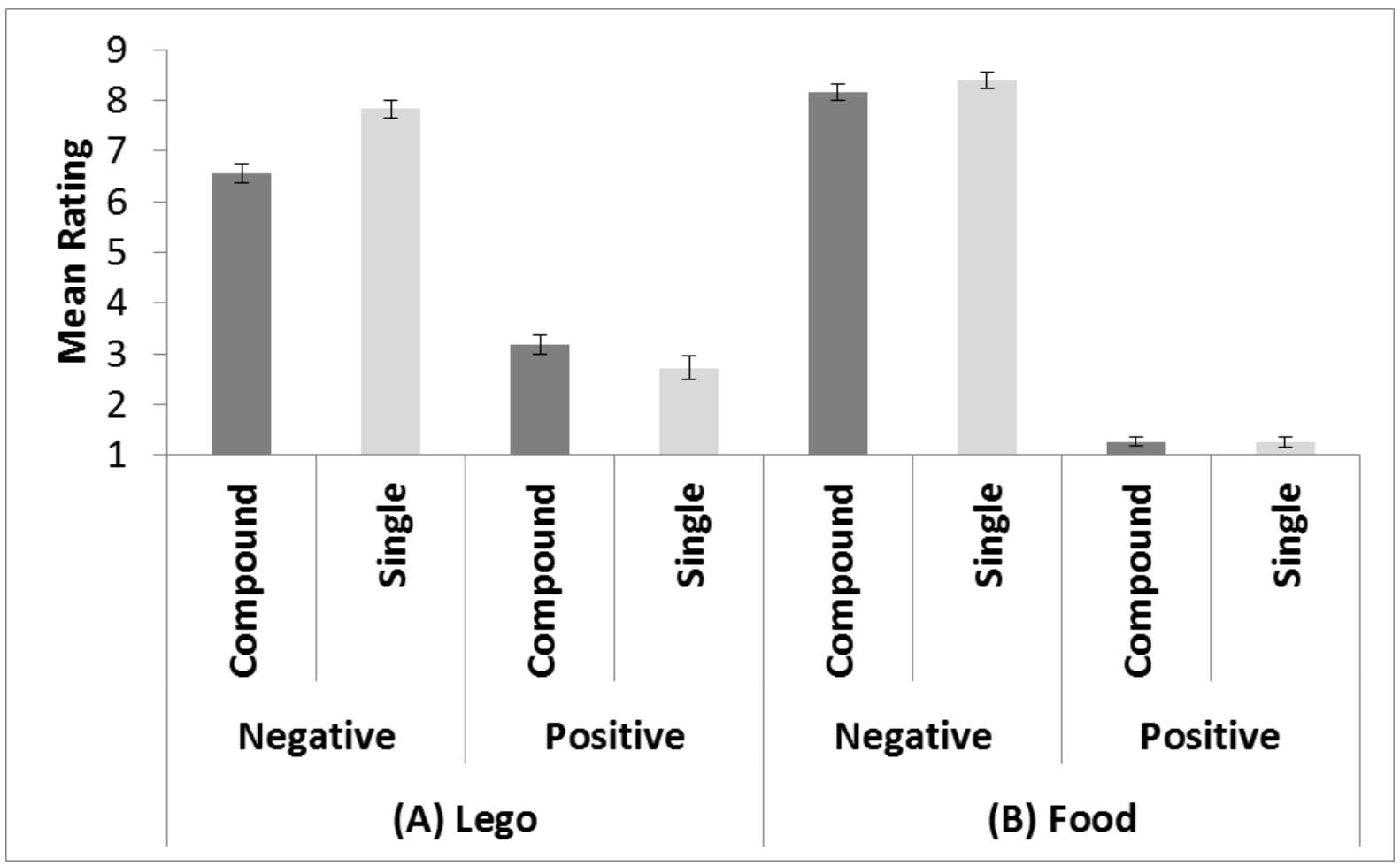

Note: Mean test ratings for (A) Lego and (B) the food allergy task variant according to presentation (compound; single) and outcome (negative; positive/'omitted') during conditioning. The rating scale was used to indicate $1=$ unlikely, $5=$ unsure, $9=$ very likely of the outcome to follow. Bars show the standard error of the mean for approximate between groups comparisons.

The three-way interaction between cue, presentation and outcome, $F(1,65)=13.569, p$ $<.001, M S e=1.293, r_{i:}^{\ddot{i}}=.173$, suggests that the level of OS was different for the Lego and food task variants, as well as for negative versus positive outcomes (see Figure 2). For the Lego cue the simple effects of outcome were significant for both the compound and single 
presentations $(p<.001)$ with mean ratings being greater for the negative cues. The simple effect of presentation was significant for negative cues $(p<.001)$ with the mean ratings for single presentation being greater than for compound presentation indicating evidence of OS. The simple effect of presentation for the positive cues was not significant ( $p=.099)$. For the food cue the simple effects of outcome were also significant for both compound and single presentations $(p<.001)$ with mean ratings again being greater for the negative cues. Unlike the Lego cue neither of the presentation simple effects were significant (negative: $p=.167$; positive: $p=.751$ ). Thus, there is no evidence of OS when using food cues.

\subsection{Correlational analyses}

These were conducted to explore the extent to which the level of OS was related to the measures of individual differences. Table 2 shows the descriptive statistics for participants' OS, BIS/BAS and schizotypy scores.

\section{Table 2}

The means and standard deviations for the OS scores, O-LIFE and BIS/BAS subscales.

\begin{tabular}{|c|c|c|c|c|c|}
\hline \multicolumn{6}{|c|}{ OS Rating Differences } \\
\hline & Lego-N & Lego-P & Food-N & \multicolumn{2}{|l|}{ Food-P } \\
\hline Mean & 1.27 & -0.45 & 0.24 & \multicolumn{2}{|l|}{-0.02} \\
\hline S.D. & 1.95 & 2.21 & 1.40 & \multicolumn{2}{|l|}{0.58} \\
\hline \multicolumn{6}{|c|}{ O-LIFE } \\
\hline & UnExp & CogDis & IntAn & \multicolumn{2}{|l|}{ ImpNo } \\
\hline Mean & 2.69 & 5.05 & 1.74 & \multicolumn{2}{|l|}{3.12} \\
\hline S.D. & 2.69 & 3.21 & 1.54 & \multicolumn{2}{|l|}{2.29} \\
\hline \multicolumn{6}{|c|}{ BIS/BAS } \\
\hline & BAS-Dr & BAS-FS & BAS-RR & FFFS & BIS-Anx \\
\hline Mean & 10.64 & 11.23 & 16.55 & 8.77 & 12.77 \\
\hline S.D. & 2.08 & 2.67 & 2.46 & 1.75 & 2.69 \\
\hline
\end{tabular}

Note: $\mathrm{N}$ indicates negative outcome and $\mathrm{P}$ indicates positive 'omitted' outcome trials in the Lego and food task variants. The O-LIFE sub-scales are unusual experiences (UnExp), cognitive disorganisation (CogDis), introvertive anhedonia (IntAn), impulsive nonconformity (ImpNo). The BIS-BAS scales are BAS Reward Responsiveness (BAS-RR), BAS FunSeeking (BAS-FS) and BAS Drive (BAS-Dr), BIS-Fight-Flight-Freeze System (BIS-FFFS) and BIS-Anxiety (BIS-Anx). 
There were no significant relationships for either task between the four O-LIFE subscales and the negative versus positive outcome OS measures for the Lego and food variants (largest $r=-.198){ }^{1}$

There was a significant positive correlation between the OS measure for the positive cues in the food task and the BAS Drive sub-scale scores, $r=.291, p=.018$. There was a significant negative correlation between the OS measure for the positive cues for the food task and the BIS-anxiety sub-scale scores, $r=-.260, p=.035$. All other correlations between the BIS/BAS and OS measures were non-significant (largest $r=-.224)$.

There was a positive correlation between CogDis scores and BIS-Anxiety, $r=.305$, $p=.013$, and BIS-FFFS scores, $r=.435, p<.001$. There was also a positive correlation between ImpNo scores and both BAS-Drive, $r=.311, p=.012$, and BIS-FFFS scores, $r=.281, p=.001$.

\section{Discussion}

The results show that learning occurred in both task variants, manifest as test expectation ratings which showed clear differentiation of cues followed by negative versus positive outcomes. In the food allergy task, contrary to prediction, there was no difference between compound and singly conditioned cues and thus no evidence of OS. In contrast OS was demonstrated in the Lego task variant. This was manifest as lower ratings for negative cues trained in compound rather than the single cue condition. Nonetheless, as Figure 2 shows, the discrimination between negative and positive outcome trials was more clearly learned for the food allergy variant.

Memory is necessary for learning and the food images used in the present study would be readily encoded in comparison with novel Lego constructions that would take longer and more effort to encode. Consistent with this suggestion, conditioning to foodrelated cues was stronger than that seen when Lego images were used as CSs. OS is a generally robust effect, the lack of any difference between cues presented singly rather than

1 Following the statistical approach used in Granger et al. [31], four stepwise regressions were carried out using the four dimensions derived from the O-LIFE and age and gender as covariates and the four OS measures as the DV. All of these regressions were not significant. This was done for the purpose of direct comparison with Granger et al [31] as normally a regression analysis is not recommended for samples of this size [47]. 
in compound in the negative outcome trials of the food allergy task may reflect a ceiling effect. Similarly, on positive outcome trials the ratings were very low, for both cues presented singly and in compound for the food allergy task. Since there was no response requirement during the conditioning stage the course of acquisition could not be examined in the present study. Possibly, evidence of OS in the food task variant might have been found had we examined earlier in the conditioning trials.

Many human associative learning tasks suffer the problem that the reinforcement is not comparable to that used in animal studies. Relatedly, it can also be difficult to specify a non-event in human associative learning studies and thus reinforcement versus nonreinforcement in the tradition of classical conditioning. We have therefore described the absence of outcome trials as positive (since the outcomes of food allergy and building collapse are unwanted) rather than non-reinforced. It must also be acknowledged that we have measured expectancy ratings rather than any more conventional conditioned response. Moreover, in common with other conditioning procedures in which humans and other animals show acquisition there is a memory component to the task. Nonetheless learning of the kind demonstrated in the present study can be related to associative learning theories developed in animals [5-7]. Indeed, Dickinson, Shanks and Evenden [48] propose that human causal learning (of the kind seen in the present tasks) develops on a trial-by-trial basis according to principles encapsulated in these theories; in other words, that cause-effect associations are learnt in the same way as CS-US associations. Differences in conditioning to food-related cues have also been identified within the animal learning literature [49] and support the concept of preparedness or relevance as a determinant of the ease with which different types of cue are associated with different kinds of reinforcer [50]. Of the more general theories of associative learning such differences are most readily accommodated by the Mackintosh theory [5] which specifically caters for the effects of past experience on associability.

An alternative position taken is that human associative learning depends on effortful, controlled reasoning processes, which necessitate propositional knowledge [51-53]. The present data provide no basis to distinguish between these accounts of human associative learning but the Lego cue variant would seem relatively unlikely to invoke controlled reasoning as it used 12 novel stimuli, about which the participants had no prior knowledge. Moreover, as (shown in Figure 1) the kinds of Lego images used were not readily identifiable by the use of objective labels, which may also provide a mechanism to facilitate learning 
[54]. Thus performance in the food allergy variant was more likely to have been influenced by participants controlled reasoning about the cue-outcome relationship, as the cues had readily available labels. Such reasoning may have been based on participants' prior knowledge as to whether particular food cues typically lead to food allergy or not. Thus higher cognitive processes may have overridden OS in this case. In contrast, the Lego task variant in which OS was demonstrated may have more in common with other human associative learning tasks which have reported an OS effect (e.g., geometric place learning $[31,55])$. In any event, the findings of the present study suggest that the presence of OS may depend on certain parameters within the design of the experiment, as appears to be the case for KB [56].

However, contrary to the hypothesis and the findings of Granger et al. [31] there was no association between the OS scores and the O-LIFE subscales, specifically UnExp, as was found in the Granger et al. [31] study or CogDis which has been linked to attentional difficulties as measured in $\mathrm{LI}$ and $\mathrm{KB}[13,29,30]$. One explanation is that the tasks used here were different from that used to show OS in Granger et al. [31]. These authors used a geometric learning task; for example two triangles presented in a composite image formed a compound stimulus in the OS condition and a single isosceles triangle for the control condition; participants were required to learn a designated 'correct corner' which remained constant over trials. Moreover, in the Granger et al. [31] task there was an instrumental component because of the feedback provided throughout conditioning: correct responses were followed by the sound of applause through the headphones. In contrast, in the present tasks, participants learning trials were observational (with no response requirement) and no feedback was given. Such methodological differences may account for the relationship between OS and O-LIFE scores shown in Granger et al. [31]. In any event, the results of the present study may be taken to suggest that the relationship between O-LIFE scores and OS is less compelling than that demonstrated for LI and KB [13, 28-30]. Consistent with this interpretation, there is evidence from animal studies that LI and KB are modulated by different neural substrates to OS [19-21]. This finding is of potential practical as well as theoretical importance because OS is a standard control in $\mathrm{KB}$ designs.

The interpretation of negative data is always difficult and one possibility for the lack of any observed relationship with the O-LIFE scores could be that reported schizotypy levels were low in the sample of participants tested. In the present study, participants' mean scores for the UnExp and IntAn sub-scales were somewhat lower than the norms reported for the 
short version of the O-LIFE based on a large twin study [24]. This difference was unlikely to be related to social desirability influences on participants' willingness to endorse sensitive items as CogDis and ImpNo scores were higher than the published population norms [24]. Granger et al. [31] used the full 104 item O-LIFE questionnaire so their participants' scores are not directly comparable with those reported here. However, the reported UnExp scores here too [31] were lower than the population norm for the full scale.

It is also possible that the present study was underpowered in the event of a small effect size [39]. However, the sample size used was similar to that reported in Granger et al. [31] (2012) as well as studies of associative learning in our laboratory which suggested that learning was related to schizotypy scores [57]. Moreover, in the present study, there were some significant relationships between O-LIFE and BIS-BAS scores. The observed correlations between the questionnaire sub-scales are broadly consistent with previous research on reinforcement sensitivity in social anxiety and schizophrenia [58-60]. For example, CogDis scores are related to cognitive difficulties with attention, concentration and decision-making. BIS-Anxiety manifests as worry and rumination and conflict resolution when avoidance-approach, avoidance-avoidance or approach-approach systems have both been activated but BIS overactivity may also be expressed at a cognitive level, as difficulties with making decisions, which could also result in attention and concentration problems.

BIS-BAS scores were also related to the behavioural OS measures. Specifically, OS scores in the food task correlated positively with BAS-Drive and negatively with BISanxiety. In each case for positive trials which represented the absence of the food allergy outcome. Higher OS scores reflect a relatively larger difference in conditioning to food CSs presented in compound versus alone [4]. In a different human associative learning task using positive IAPS images as USs, higher BAS-Drive scores have been found to predict reduced excitatory conditioning whereas higher BIS-Anxiety scores showed a different profile, predicting increased inhibitory learning [39]. In the present study, the relationships with BISBAS were shown only for the data from the positive 'omission' trials which maybe may be more akin to inhibitory learning but which also in effect provide an alternative (motivationally opponent) outcome representation ('no allergy'). BIS was originally postulated to be activated by signals of non-reward [34, 61] and, according to revised RST, BIS-Anxiety mediates the processing of conflict-related cues [35, 62]. At a more general level, the negative relationship between BIS-Anxiety and OS suggests that OS diminished with increased trait anxiety. This is consistent with evidence which suggests trait anxiety may 
enhance cognitive performance [63]. Moreover, anxious individuals also show greater attentional orientation towards safety and 'relieving' cues [64]. Thus, perhaps the 'no allergy' outcome may have been perceived as a safety signal, hence enhanced attention and learning in the positive outcome condition for participants higher in trait anxiety.

Food allergy paradigms are commonly adopted in human causal learning tasks but may not be ideal measures of all aspects of associative learning and its related phenomena, because they typically present participants with familiar cues with pre-existing associations. The results of the present study show that the overall OS effect (manifested as better conditioning to negative cues presented singly rather than in compound) was only demonstrated in a formally similar Lego task variant which used more neutral (less familiar) CSs than can be provided by food images. Nonetheless, some associations between the level of OS and reinforcement sensitivity as measured by the BIS-BAS were demonstrated in the positive outcome condition of the food allergy task (only). Thus the development of OS was found to depend on cue and reinforcement sensitivity. In particular, the differential association with BIS-BAS scores found in the present study suggests that task variant is a determinant of the brain substrates of OS. Further studies will be necessary to delineate the boundary conditions for (negative) relationships between schizotypy and selective learning based on cue salience. 


\section{References}

[1] Lubow, R. E., \& Moore, A. U. (1959). Latent inhibition: the effect of nonreinforced preexposure to the conditional stimulus. Journal of Comparative and Physiological Psychology, 52 (September), 415-419.

[2] Kamin, L.J. (1969). Predictability, surprise, attention and conditioning. In Campbell, B.A. \& Church, R.M. (Eds.), Punishment and Aversive Behaviour. (pp. 279-296). NY: Appleton Century Crofts.

[3] Cassaday, H. J. \& Moran, P. M. (2010). Latent inhibition and other salience modulation effects: same neural substrates? In Lubow, R. \& Weiner, I. (Eds.), Latent Inhibition: Cognition, Neuroscience, and Applications to Schizophrenia (pp.342-371). Cambridge University Press.

[4] Pavlov, I. P. (1927). Conditioned Reflexes. Oxford University Press.

[5] Mackintosh, N. J. (1975). A theory of attention: variations in the associability of stimuli with reinforcement. Psychological Review, 82(4), 276-298.

[6] Pearce, J. M., \& Hall, G. (1980). A model for Pavlovian learning: variations in the effectiveness of conditioned but not of unconditioned stimuli. Psychological Review, $87(6), 532-552$.

[7] Rescorla, R.A. \&Wagner A.R. (1972). A theory of Pavlovian conditioning: variations in the effectiveness of reinforcement and nonreinforcement. In Black A.H. \& Prokasy W.F. (Eds.), Classical Conditioning II: Current Research and Theory (pp.64-99). New York: Appleton-Century-Crofts.

[8] Kumari, V. \& Ettinger, U. (2010). Latent inhibition in schizophrenia and schizotypy: a review of the empirical literature. In Lubow, R. \& Weiner, I. (Eds.), (pp.419-447). Cambridge University Press.

[9] Baruch, I., Hemsley, D.R., \& Gray, J. A. (1988). Differential performance of acute and chronic schizophrenics in a latent inhibition task. The Journal of Nervous and Mental Disease, 176(10), 598-606.

[10] Gray, N. S., Hemsley, D. R., \& Gray, J. A. (1992). Abolition of latent inhibition in acute, but not chronic, schizophrenics. Neurology Psychiatry and Brain Research, 1(2), 83-89.

[11] Rascle, C., Mazas, O., Vaiva, G., Tournant, M., Raybois, O., Goudemand, M., \& 
Thomas, P. (2001). Clinical features of latent inhibition in schizophrenia. Schizophrenia Research, 51(2-3), 149-161.

[12] Jones, S. H., Hemsley, D., Ball, S., \& Serra, A. (1997). Disruption of the Kamin blocking effect in schizophrenia and in normal subjects following amphetamine. Behavioural Brain Research, 88(1), 103-114.

[13] Moran, P. M., Al-Uzri, M. M., Watson, J., \& Reveley, M. A. (2003). Reduced Kamin blocking in non paranoid schizophrenia: associations with schizotypy. Journal of Psychiatric Research, 37(2), 155-163.

[14] Jones, S. H., Gray, J. A., \& Hemsley, D. R. (1992a). Loss of the Kamin blocking effect in acute but not chronic schizophrenics. Biological Psychiatry, 32(9), 739-755.

[15] Bleuler, E. (1911). Dementia Praecox or the Group of Schizophrenias. Oxford, England: International Universities Press.

[16] Howes, O. D. \& Kapur, S. (2009). The dopamine hypothesis of schizophrenia: version III - the final common pathway. Schizophrenia Bulletin, 35(3), 549-562.

[17] Kapur, S. (2003). Psychosis as a state of aberrant salience: a framework linking biology, phenomenology, and pharmacology in schizophrenia. American Journal of Psychiatry, 160(1), 13-23.

[18] Kapur, S., Mizrahi, R., \& Li, M. (2005). From dopamine to salience to psychosis-linking biology, pharmacology and phenomenology of psychosis. Schizophrenia Research, 79(1), 59-68.

[19] O’Tuathaigh, C. M. P. \& Moran, P. M. (2002). Evidence for dopamine D1 receptor involvement in the stimulus selection task: overshadowing in the rat. Psychopharmacology, 162(3), 225-231.

[20] O’Tuathaigh, C. M. P., Salum, C., Young, A. M. J., Pickering, A. D., Joseph, M. H., \& Moran, P. M. (2003). The effect of amphetamine on Kamin blocking and overshadowing. Behavioural Pharmacology, 14(4), 315-322.

[21] Nelson, A.J.D., Thur, K.E., Marsden, C.A. \& Cassaday, H.J. (2011). Dopamine in nucleus accumbens: salience modulation in latent inhibition and overshadowing. Journal of Psychopharmacology, 25, 1649-1660.

[22] Mason, O. \& Claridge, G. (2006). The Oxford-Liverpool Inventory of Feelings and 
Experiences (O-LIFE): further description and extended norms. Schizophrenia Research, 82(2-3), 203-211.

[23] Mason, O., Claridge, G. \& Jackson, M. (1995). New scales for the assessment of schizotypy. Personality and Individual Differences, 18(1), 7-13.

[24] Mason, O., Linney, Y. \& Claridge, G. (2005). Short scales for measuring schizotypy. Schizophrenia Research, 78(2-3), 293-296.

[25] Barrantes-Vidal N., Grant P. \& Kwapil T.R. (2015). The role of schizotypy in the study of the etiology of schizophrenia spectrum disorders. Schizophrenia Bulletin, 41 (Suppl. 2), S408-S416.

[26] Everett, K.V. \& Linscott, R.J. (2015). Dimensionality vs taxonicity of schizotypy: some new data and challenges ahead Schizophrenia Bulletin, 41 (Suppl. 2), S465-S474.

[27] Claridge, G. (1987). 'The schizophrenias as nervous types' revisited. The British Journal of Psychiatry, 151(6), 735-743.

[28] Evans, L. H., Gray, N. S., \& Snowden, R. J. (2007). A new continuous withinparticipants latent inhibition task: examining associations with schizotypy dimensions, smoking status and gender. Biological Psychology, 74(3), 365-373.

[29] Gray, N. S., Fernandez, M., Williams, J., Ruddle, R. A., \& Snowden, R. J. (2002). Which schizotypal dimensions abolish latent inhibition? The British Journal of Clinical Psychology, 41(3), 271-284.

[30] Jones, S. H., Gray, J. A., \& Hemsley, D. R. (1992b). The Kamin blocking effect, incidental learning and schizotypy (a reanalysis). Personality and Individual Differences, 13(1), 57-60.

[31] Granger, K. T., Prados, J., \& Young, Q. M. J. (2012). Disruption of overshadowing and latent inhibition in high schizotypy individuals. Behavioural Brain Research, 233(1), 201-208.

[32] Granger, K. T., Moran, P.M., Buckley, M.G., \& Haselgrove M. (2016). Enhanced latent inhibition in high schizotypy individuals. Personality and Individual Differences, 91, $31-39$.

[33] Gray, J. A. (1970). The psychophysiological basis of introversion-extraversion. Behaviour Research and Therapy, 8(3), 249-266. 
[34] Gray, J. A. (1982). The Neuropsychology of Anxiety: An Enquiry into the Functions of the Septo-Hippocampal System. Oxford University Press.

[35] Gray, J. A. \& McNaughton, N. (2000). The Neuropsychology of Anxiety: An Enquiry into the Functions of Septo-hippocampal System, 2nd Ed. Oxford University Press.

[36] Carver, C. S., \& White, T. L. (1994). Behavioral inhibition, behavioral activation, and affective responses to impending reward and punishment: the BIS/BAS scales. Journal of Personality and Social Psychology, 67(2), 319.

[37] Corr, P. J. (2008). Reinforcement Sensitivity Theory (RST): Introduction. In Corr, P. J. (Ed.). The Reinforcement Sensitivity Theory of Personality. (pp.1-43). Cambridge University Press.

[38] Heym, N., Ferguson, E., \& Lawrence, C. (2008). An evaluation of the relationship between Gray's revised RST and Eysenck's PEN: distinguishing BIS and FFFS in Carver and White's BIS/BAS scales. Personality and Individual Differences, 45(8), 709715.

[39] He, Z., Cassaday, H. J., Bonardi, C., \& Bibby, P. A. (2013). Do personality traits predict individual differences in excitatory and inhibitory learning? Frontiers in Psychology, 4(May), 1-12.

[40] Dickinson, A. (2001). The 28th Bartlett Memorial Lecture. Causal learning: an associative analysis. The Quarterly Journal of Experimental Psychology. B, Comparative and Physiological Psychology, 54(1), 3-25.

[41] Waldmann, M. (2001). Predictive versus diagnostic causal learning: evidence from an overshadowing paradigm. Psychonomic Bulletin \& Review, 8(3), 600-608.

[42] Waldmann, M., \& Walker, J. (2005). Competence and performance in causal learning. Learning \& Behavior, 33(2), 211-229.

[43] Vandorpe, S., \& Houwer, J. De. (2006). A comparison of cue competition in a simple and a complex design. Acta Psychologica, 122(3), 234-246.

[44] Van Hamme, L. J., \& Wasserman, E. A. (1994). Cue competition in causality judgments: the role of nonpresentation of compound stimulus elements. Learning and Motivation, 25(2), 127-151.

[45] Vogel, E. H., Glynn, J. Y., \& Wagner, A. R. (2015). Cue competition effects in human 
causal learning. The Quarterly Journal of Experimental Psychology, 68(12), 2327-2350.

[46] Peirce, J.W. (2009). Generating stimuli for neuroscience using PsychoPy. Frontiers in Neuroinformatics. 2(January), Article 10.

[47] Field, A. (2013). Discovering Statistics Using IBM SPSS Statistics. Sage.

[48] Dickinson, A., Shanks, D., \& Evenden, J. (1984). Judgement of act-outcome contingency: the role of selective attribution. The Quarterly Journal of Experimental Psychology, 36(1), 29-50.

[49] Garcia, J., \& Koelling R.A. (1966). Relation of cue to consequence in avoidance learning. Psychonomic Science 4(1), 123-124.

[50] Seligman M.E.P. (1970). On the generality of the laws of learning. Psychological Review, 77(5), 406-418.

[51] De Houwer, J., Beckers, T., \& Vandorpe, S. (2005). Evidence for the role of higher order reasoning processes in cue competition and other learning phenomena. Learning \& Behavior, 33(2), 239-249.

[52] Mitchell, C. J., De Houwer, J., \& Lovibond, P. F. (2009). The propositional nature of human associative learning. Behavioral and Brain Sciences, 32(2), 183-198.

[53] Shanks, D. R. (2010). Learning: from association to cognition. Annual Review of Psychology, 61, 273-301.

[54] Lupyan, G., Rakison, D.H., \& McClelland, J.L. (2007). Language is not just for talking: Redundant labels facilitate learning of novel categories. Psychological Science, 18(12), 1077-1083.

[55] Prados, J. (2011). Blocking and overshadowing in human geometry learning. Journal of Experimental Psychology. Animal Behavior Processes, 37(1), 121-126.

[56] Maes, E., Boddez, Y., Alfei, J. M., Krypotos, A.-M., D’Hooge, R., De Houwer, J., \& Beckers, T. (2016). The elusive nature of the blocking effect: 15 failures to replicate. Journal of Experimental Psychology: General. Advance online publication. http://dx.doi.org/10.1037/xge0000200

[57] Migo, E.M., Corbett, K., Graham, J., Smith, S., Tate, S., Moran, P.M. \& Cassaday, H.J. (2006). A novel test of conditioned inhibition correlates with personality measures of schizotypy and reward sensitivity. Behavioural Brain Research, 168(2), 299-306. 
[58] Horan, W. P., Wynn, J. K., Mathis, I., Miller, G. A., \& Green, M. F. (2014). Approach and withdrawal motivation in schizophrenia: an examination of frontal brain asymmetric activity. PLoS ONE, 9(10), e110007.

[59] Kimbrel, N. A., Nelson-Gray, R. O., \& Mitchell, J. T. (2012). BIS, BAS, and bias: the role of personality and cognitive bias in social anxiety. Personality and Individual Differences, 52(3), 395-400.

[60] Scholten, M. R.M., van Honk, J., Aleman, A., \& Kahn, R. S. (2006). Behavioral inhibition system (BIS), behavioral activation system (BAS) and schizophrenia: relationship with psychopathology and physiology. Journal of Psychiatric Research, 40(7), 638-645.

[61] Gray, J. A. (1972). The psychophysiological basis of introversion-extraversion: a modification of Eysenck's theory. In V. D. Nebylitsyn \& J. A. Gray (Eds.), The Biological Bases of Individual Behaviour (pp.182-205). San Diego, CA: Academic Press.

[62] Corr, P. J. (2010). The psychoticism-psychopathy continuum: a neuropsychological model of core deficits. Personality and Individual Differences, 48(6), 695-703.

[63] Perkins, A. M., \& Corr, P. J. (2005). Can worriers be winners? The association between worrying and job performance. Personality and Individual Differences, 38(1), 25-31.

[64] Derryberry, D., \& Reed, M. A. (2002). Anxiety-related attentional biases and their regulation by attentional control. Journal of Abnormal Psychology, 111(2), 225-236. 